\title{
Exploring meal frequency and vegetable intake among immigrants 70 years or older in Sweden
}

\author{
Secondary analysis of data from the Promoting Aging Migrants Capabilities study \\ Julie Johannesson ${ }^{1,2,3^{*}} \mathbb{D}$, Susanne Gustafsson ${ }^{3,4}$ (D) Frode Slinde Fi, $^{1,5}$ and Elisabet Rothenberg ${ }^{6}$ (D)
}

\begin{abstract}
Background: Earlier research regarding immigrants has shown that lifestyle and environmental factors are of importance for the risk of developing certain diseases. Food habits are one of these factors. As there is little research concerning immigrants and food habits, the aim of the present paper was to explore food habits operationalised as meal frequency and vegetable intake among a group of older immigrants in Sweden. Specifically, the following questions were explored: changes in food habits when migrating, missing food from the native country, gender differences in food habits and association between food habits and self-rated health and well-being.
\end{abstract}

Method: A total of 131 persons from the health-promoting study "Promoting Aging Migrants' Capabilities" (PAMC) conducted in Gothenburg, Sweden, 2011-2014, were included in this exploratory secondary analysis of questionnaire data. Participants were 70 years or older, cognitively intact, and living in ordinary housing. They had all migrated to Sweden from Finland or the Western Balkan Region (Bosnia-Herzegovina, Croatia, Montenegro and Serbia). Descriptive statistics as well as binary logistic regression were used to answer the research questions.

Results: Forty-eight percent of the participants in PAMC reported that they had changed their food habits when migrating, and $17 \%$ considered that they missed certain foods from their native country. Most of the participants migrated to Sweden more than 20 years ago. There was a significant difference in self-rated health in favour of the male participants $(p=0.02)$, but food habits, operationalised as meal frequency and vegetable intake, were not associated with self-rated health or life satisfaction.

Conclusion: Men rated their health as better than women did, but food habits operationalised as meal frequency or vegetable intake, were not associated with self-rated health or life satisfaction. Results from this secondary analysis were affected by methodological shortcomings. Further studies to understand the role of food habits in relation to health in a migration context are desired.

Trial registration: NCT01841853, Registered April 29, 2013. Retrospectively registered.

Keywords: Older adults, Immigrants, Meal frequency, Vegetable intake, Self-rated health, Life satisfaction, Exploratory study, Secondary analysis

\footnotetext{
*Correspondence: julie.johannesson@gu.se

${ }^{1}$ Department of Internal Medicine and Clinical Nutrition, Sahlgrenska

Academy at University of Gothenburg, P.O. Box 459, 40530 Gothenburg, Sweden

Full list of author information is available at the end of the article
}

\section{Introduction}

In an increasingly globalised world, many people, for different reasons, move from one country to another during their lifetime. In 2019 , about $20 \%$ of the total population original author(s) and the source, provide a link to the Creative Commons licence, and indicate if changes were made. The images or other third party material in this article are included in the article's Creative Commons licence, unless indicated otherwise in a credit line to the material. If material is not included in the article's Creative Commons licence and your intended use is not permitted by statutory regulation or exceeds the permitted use, you will need to obtain permission directly from the copyright holder. To view a copy of this licence, visit http://creativecommons.org/licenses/by/4.0/. 
of approximately 10 million in Sweden were first-generation immigrants (i.e. born outside Sweden). By 2060, it is estimated that a quarter of the older population ( $\geq 80$ years) will have a background as an immigrant [1]. In 2019, 31\% of immigrants aged 70 years and older in Sweden were born in Finland and 4\% in the Western Balkan region (Bosnia-Herzegovina, Croatia, Montenegro and Serbia) [2].

There is little research concerning older immigrants' food habits in Sweden, and therefore, the knowledge gap is significant. In early 1990s, Kochtürk-Runefors and co-workers [3] stated that immigrants constitute large groups of people who make major changes to their diets during a lifetime. The adaptation and acculturation (the process where immigrants adopt the dietary practices of their new country [4]) that migration involves have an important impact on behavioural factors such as food habits [5]. Length of time since migration [6] has not been associated with a poorer quality of life among older immigrants ( $>60$ years), but a strong association between people who have experienced migration and the prevalence of cardiovascular disease has been found [6].

Self-rated health has been shown as a reliable measure of the overall health and is also proven to be a significant predictor for the development of morbidity, mortality and disability in activities of daily living (ADL) among older adults [7]. Another important estimate of health is life satisfaction, which could be defined as "an endorsement of, or positive attitude toward one's life overall" [8]. Levels of life satisfaction are among other factors affected by health status. Since older adults are likely to experience a decline in physical health and functional ability, this assessment has shown to be especially important among the very old ( $>85$ years) [9]. In a recent Norwegian study [10] concerning persons $>65$ years, it was found that life satisfaction and mental health was associated with higher scores on life satisfaction. A report from Gothenburg municipality shows that there are differences in self-rated health between people born in Sweden and those born outside Sweden; people born outside Sweden rate their health to a greater extent as worse than those born in Sweden. Inequality in health has increased over the last two decades for foreign-born people [11].

In a substudy [12] to the randomised controlled trial Elderly Persons in the Risk Zone (EPiR) [13], it was shown that in a group of older adults ( $>80$ years), habits regarding cooking, meal patterns and choice of foods changed when getting older, mainly because of retirement or losing a spouse. Women took a greater responsibility for planning food, shopping and cooking, and a large proportion of men thought that cooking was something they were not able to do [12]. EPiR was a predecessor to the study Promoting Aging Migrants' Capabilities (PAMC)
[14], a randomised controlled trial among $\geq 70$ years who have experienced migration from Finland or the Western Balkan region. The hypothesis tested in PAMC was that senior meetings would prevent and delay deterioration in health (i.e. dependence in ADL, self-rated health) and maintain life satisfaction among older persons born abroad. These senior meetings have a person-centred approach to health promotion in order to support optimal ageing and were found to have positive effects in the preceding EPiR study [15]. For more information about EPiR and PAMC, please see the study protocols $[13,14]$.

In the present paper, we explore food habits, operationalised as meal frequency and vegetable intake, among participants at baseline in a secondary analysis of PAMC [14]. As there is little research concerning immigrants and health-related factors such as food habits, the following research questions were addressed:

- Have participants changed their food habits after migrating to Sweden?

- Do participants miss any food from their native country?

- Are there any gender differences in food habits, selfrated health, and life satisfaction?

- Are there any associations between food habits and self-rated health and life satisfaction?

\section{Design and methods}

This cross-sectional study is an exploratory [16] secondary analysis for which baseline data from the randomised controlled trial PAMC is used [14]. An exploratory approach is a preferable choice when little is known about the studied phenomenon, as is the case for food habits among older immigrants.

\section{Participants}

Participants in PAMC were recruited between 2011 and 2014 [14]. Finally, 131 persons aged 70-84 years were included; see Fig. 1. Inclusion criteria for PAMC were independently living persons, aged 70 years or older who had migrated to Sweden from Finland or from the Western Balkan region (Bosnia-Herzegovina, Croatia, Montenegro and Serbia). The intention with PAMC was that the study group should comprise a representative sample of older persons in the urban district who have migrated to Sweden. Particularly considering the need for language resources in terms of bilingual project assistants, persons from, at that time, the two of the largest immigrant groups in the targeted urban district were chosen: one Nordic country (Finland) and one European region (The Balkan Peninsula) [14]. Impaired cognition was the single exclusion criteria, defined as below $80 \%$ of the 


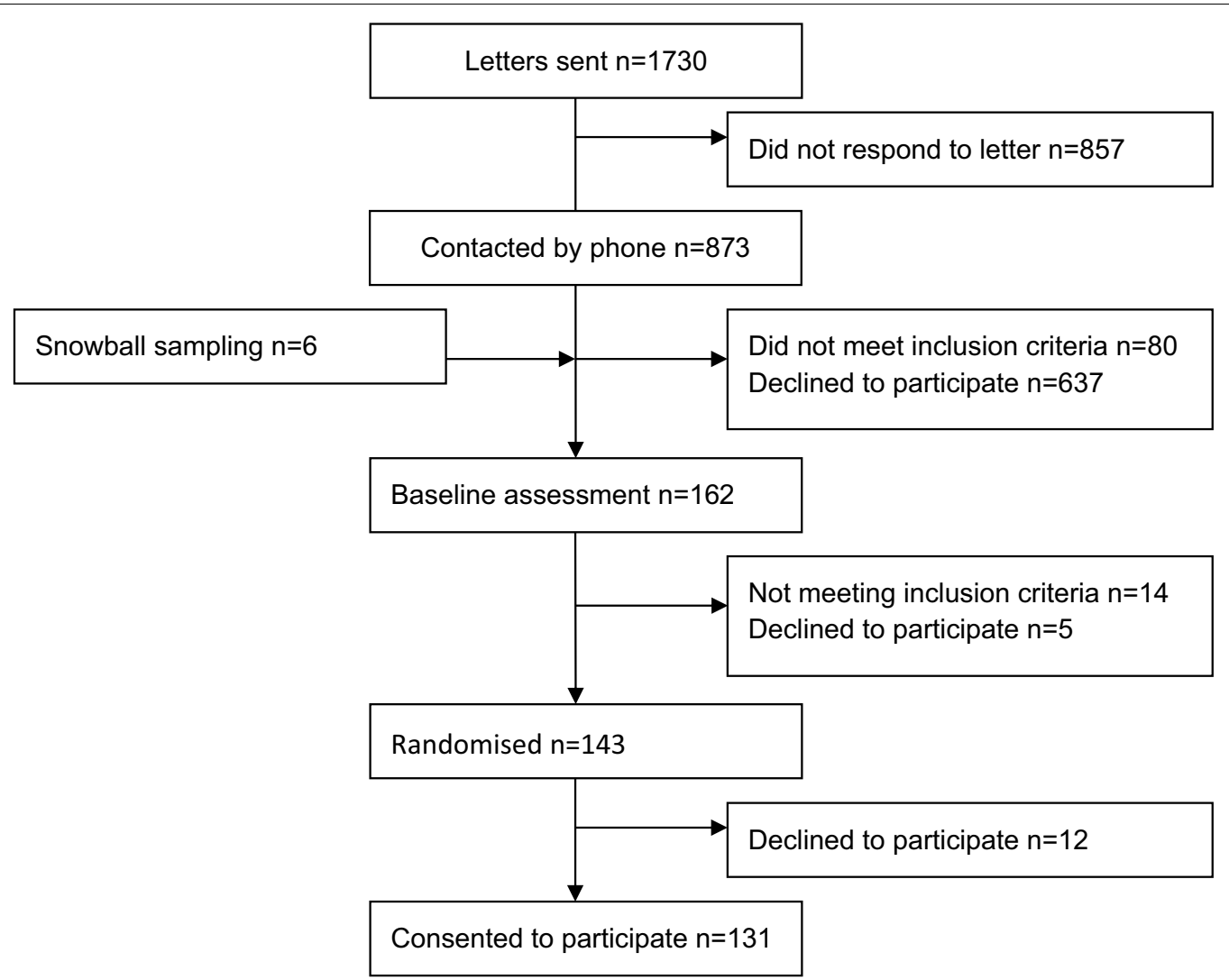

Fig. 1 The flow of participants through Promoting Aging Migrants' Capabilities study

administered items on the Mini Mental State Examination (MMSE) [17]. Further details are found in the study protocol [14].

\section{Data collection in PAMC}

In PAMC, bilingual research assistants, who had all received training by the research group on how to administer the study questionnaire, conducted semi-structured individual interviews to collect data on demographic variables and outcome measures, including the outcomes at baseline used in this paper. All interviews were conducted in the participants' choice of language and location. Follow-ups were held at 6 months and 12 months after the health-promoting intervention. The present paper used baseline data completed in December 2014 as a basis for the exploratory secondary analysis regarding food habits operationalised as meal frequency and vegetable intake.

\section{Methods}

A detailed description of the items used for measurements are presented in Fig. 2. Two questions regarding food habits after migrating were asked. In order to answer the third and fourth research questions a set of measures were used: self-rated health, assessed by the first overall question in the 36 Item Short Form Health Survey (SF-36) [18], and life satisfaction for which the validated LiSat-11 scale was used [19]. The original scale consists of eleven items. In this paper eight items were used; assessing satisfaction with life as a whole as well as satisfaction with work, financial situation, leisure activities, friends and acquaintances, functional capacity, physical health and physiological health. The questions concerning marital status, sexual life and family life were removed as the reference groups in the original study, consisting of older persons from Finland and the Western Balkan region, considered the questions too sensitive for the targeted group.

\section{Possible confounding variables}

Seven items commonly regarded as confounding in the relation to food habits [20] were considered, see Fig. 2. Median age was calculated and age was dichotomised into 70-74 years and 75-84 years with median age as cut-off. Participants with an academic degree or an incomplete academic education were dichotomised as "tertiary education". Participants not living with a cohabitant or spouse were dichotomised as "living alone". 


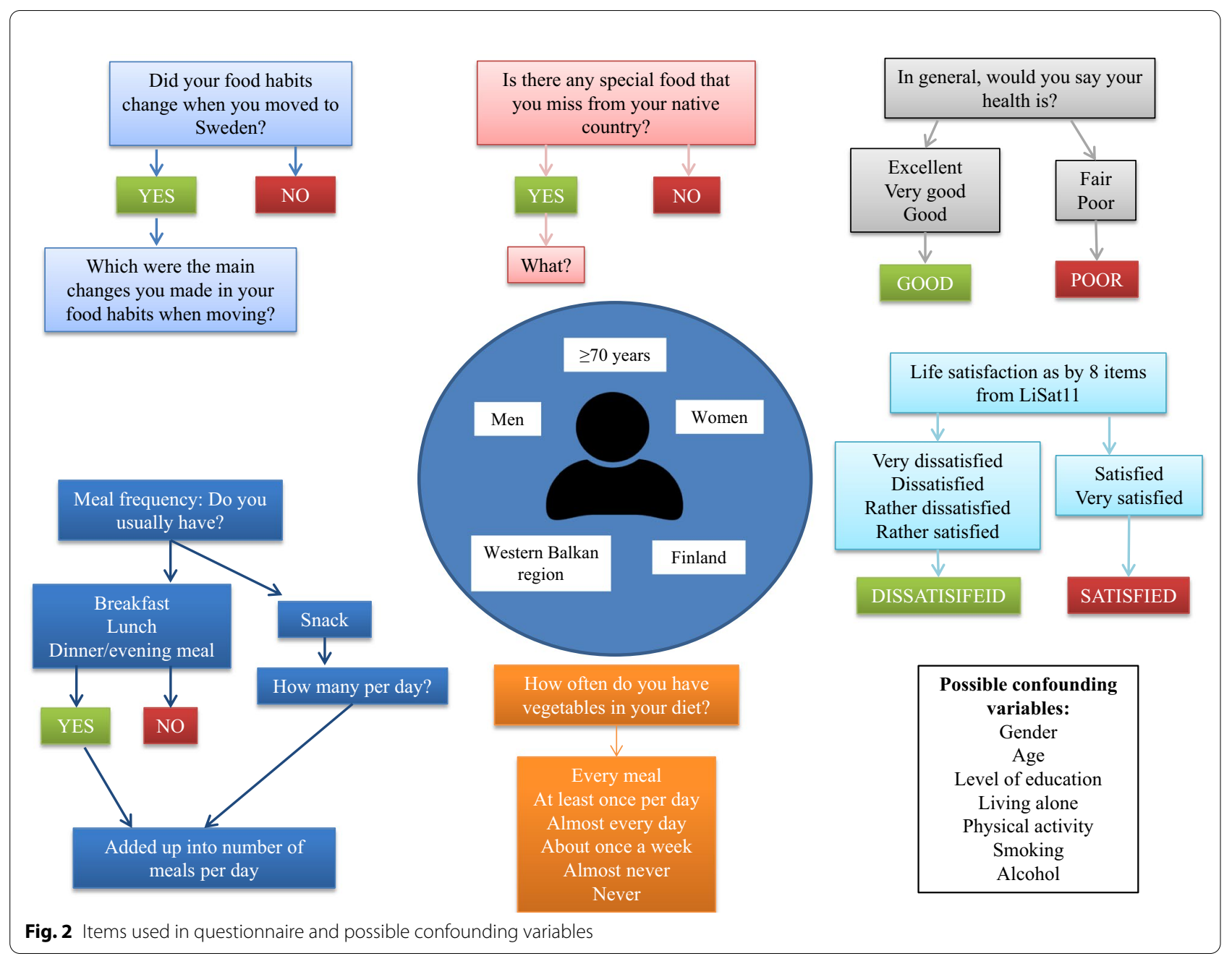

Those who went for a walk at least four times a week or more were dichotomised as "high physical activity". Participants were asked if they were smokers, and they were also asked how often they consumed alcohol; never, once a month or more seldom, 2-4 times per month, 2-3 times per week or 4 times per week or more. Response alternatives were dichotomised into "never" (never or once a month or more seldom) or " $2-4$ times per month or more" (2-4 times per month, $2-3$ times per week or 4 times per week or more).

\section{Statistical analysis}

The descriptive results are presented as proportions, mean and standard deviation (SD) when applicable. Meal frequency was operationalised as breakfast, lunch, dinner and snack and the stated number of meals were added up with number of snacks into total per day. In the regression analysis, dependent variables were self-rated health and life satisfaction. Food habits constituted the independent variable and were operationalised into two sub variables; meal frequency and vegetable intake. To analyse differences between gender in meal frequency, vegetable intake, self-rated health and life satisfaction, the Chi2 test was used and statistical significance was accepted at the $5 \%$ level $(p<0.05)$. Binary logistic regression was utilised to examine the associations between meal frequency and self-rated health and life satisfaction, respectively, and between vegetable intake and self-rated health and life satisfaction, respectively. All statistical analyses were performed using IBM SPSS Statistics, version 24.0 (IBM Corp., Armonk, NY, USA, 2016).

\section{Ethical considerations}

The Regional Ethical Review Board in Gothenburg approved PAMC (\#821-11) and all participants received written and oral information that participation was completely voluntary and that they could decline at any time without giving a reason. All written and oral information for informed consent in PAMC was given in the participants' preferred language to ensure that they were 
aware of what participation entailed. The PAMC was registered at ClinicalTrials.gov April 10, 2013, identifier: NCT01841853.

\section{Results}

\section{Study population characteristics}

A total of 131 community-dwelling persons, 50\% women $(n=65)$, aged between 70 and 84 years who participated at baseline in PAMC were included in this secondary analysis. Fifty-four percent $(n=71)$ were born in Finland, $46 \%(n=60)$ in the Western Balkan region. A majority $(87 \%, n=114)$ had lived in Sweden for more than 20 years. Further characteristics are presented in Table 1.

\section{Food habits}

Forty-eight percent $(n=63)$ believed that their food habits had changed since moving to Sweden. The major changes were that bread, potatoes, rice, pasta, fruit and vegetable intake was reported as increased, compared to

Table 1 Baseline characteristics of the study population $(n=131)$

\begin{tabular}{|c|c|}
\hline Variable & Total $n=131$ \\
\hline Female, $n(\%)$ & $65(50)$ \\
\hline Age, mean, $\pm S D$, range & $74 \pm 3.4,(70-84)$ \\
\hline \multicolumn{2}{|l|}{ Age, by groups, $n(\%)$} \\
\hline $70-74$ & $68(52)$ \\
\hline $75-84$ & $63(48)$ \\
\hline \multicolumn{2}{|l|}{ Education level, $n$ (\%) } \\
\hline Tertiary education ${ }^{\mathrm{a}}$ & $20(15)$ \\
\hline Low education ${ }^{\mathrm{b}}$ & $109(83)$ \\
\hline Did not specify & $2(2)$ \\
\hline Living alone, $n(\%)$ & $64(49)$ \\
\hline \multicolumn{2}{|l|}{ Native country, n (\%) } \\
\hline Finland & $71(54)$ \\
\hline Western Balkan region & $60(46)$ \\
\hline \multicolumn{2}{|l|}{ Years lived in Sweden, $n$ (\%) } \\
\hline $6-10$ & $1(1)$ \\
\hline $11-15$ & $1(1)$ \\
\hline $16-20$ & $15(11)$ \\
\hline$>20$ & $114(87)$ \\
\hline \multicolumn{2}{|l|}{ Type of housing, $n(\%)$} \\
\hline Rented flat & $68(52)$ \\
\hline Owner of apartment & $26(20)$ \\
\hline Owner of house & $35(27)$ \\
\hline Other & $2(1)$ \\
\hline Smoker, $n(\%)$ & $8(6)$ \\
\hline Alcohol > 2-4 times/month, $n(\%)$ & $59(45)$ \\
\hline
\end{tabular}

SD standard deviation

${ }^{\text {a }}$ Tertiary education (university $\geq 3$ years)

${ }^{\mathrm{b}}$ Low education (elementary school or no education) before migrating. Those $(n=29)$ who considered their food habits as changed, answered "other" which could include, for example, becoming a vegetarian, more salt, less salt, had to start cooking by myself, more alcohol or being able to get food at all. Some persons also answered that they changed their food habits, but they did not specifically remember how, as it was a long time since they migrated.

Seventeen percent $(n=22)$ considered that they missed certain foods from their native country that were not available in Sweden, and exemplified the following: Memma (a traditional Finnish dessert), specific dairy products, and different types of bread.

\section{Self-rated health and life satisfaction}

Sixty-eight percent $(n=89)$ considered their health as good, $77 \%(n=51)$ of the men and $59 \%(n=38)$ of the women, with a significant difference between genders $(p=0.02)$ in favour of male participants. Concerning life satisfaction, $65 \%(n=42)$ of men and $53 \%(n=34)$ of women claimed that they were satisfied with their life situation, as total $59 \%$. No significant difference between genders was found $(p=0.19)$.

\section{Meal frequency}

Concerning meal frequency, $25 \%$ of men and $38 \%$ of women had three meals per day, and $55 \%$ of men and $56 \%$ of women had four or more meals per day, including snacks. Men tended to have a higher meal frequency than women $(p=0.05)$. There was no significant association found between meal frequency and self-rated health $(p=0.59)$ (OR 1.10, CI 0.78-1.55). Adjusting for gender, age, education, living alone, level of physical activity, smoking and alcohol did not affect the result (Additional file 1). Nor was there any significant association between meal frequency and life satisfaction $(p=0.33$ ) (OR 0.85 , CI 0.60-1.18). Adjusting for gender, age, education, living alone, level of physical activity, smoking and alcohol did not affect the result (Additional file 1).

\section{Vegetable intake}

Almost half of the women $(48 \%)$ and a third $(36 \%)$ of the men claimed that they had vegetables with every meal. Five men and seven women ate vegetables seldom or never (Table 2). No significant difference between genders was found $(p=0.32)$. Nor was there any significant association between vegetable intake and self-rated health $(p=0.34)$ (OR 1.19, CI 0.84-1.68). Adjusting for gender, age, education, living alone, level of physical activity, smoking and alcohol did not affect the result (Additional file 2). Neither was a significant association found between vegetable intake and life satisfaction $(p=0.09)$ (OR 1.35, CI 0.96-1.90). Adjusting for gender, 
Table 2 Frequency of vegetable intake among women and men

\begin{tabular}{lcl}
\hline & $\begin{array}{l}\text { Men, } \boldsymbol{n} \text { (\%) } \\
\text { Total } \boldsymbol{n = 6 4}\end{array}$ & $\begin{array}{l}\text { Women, } \boldsymbol{n} \text { (\%) } \\
\text { Total } \boldsymbol{n}=\mathbf{5 9}\end{array}$ \\
\hline Every meal & $23(36)$ & $31(48)$ \\
At least once per day & $21(33)$ & $19(29)$ \\
Almost every day & $15(23)$ & $8(12)$ \\
Now and then during a week & $3(5)$ & $6(9)$ \\
Never & $2(3)$ & $1(2)$ \\
\hline
\end{tabular}

age, education, living alone, level of physical activity, smoking and alcohol did not affect the result (Additional file 2).

\section{Discussion}

The result showed that almost half of the participants in PAMC had changed their food habits, and about onefifth missed specific foods from their native country. In addition, a significant difference in self-rated health in favour of male participants was identified, but food habits, operationalised as meal frequency and vegetable intake, were not associated with self-rated health or life satisfaction. However, several persons claimed that they had changed food habits but did not remember in what way, which might be explained by the fact that a majority, almost 90\% had lived in Sweden for more than 20 years. This may imply that individuals more or less subconsciously change food habits over time. It would have been interesting to compare dietary patterns for the study participants with a comparable group of older persons born in Sweden. Unfortunately, no such data is available from the present study; however, data from a population-based sample of 70-year old adults conducted in Gothenburg showed that dietary patterns have changed and improved over time in terms of an increase in vegetable intake [21]. Satia-Abouta [4] showed that longer residence in the new country results in increased exposure to mainstream culture and with that, acculturation including food habits. It has also been shown that high education and income, employment outside the home, having children and being able to speak the new language fluently are also factors that affect acculturation. Participants in PAMC had various reasons for migration, mainly due to war or as labour migrants, which means that age when migrating differed, which could also have influenced the acculturation process differently. Preparing and eating food from one's native country plays an important role for many immigrants. The taste of home foods may further release and decrease one's feelings of nostalgia and attach a sense of belonging to a particular place [22, 23]. An earlier study on Chinese immigrants in the USA and Canada showed that older individuals preferred a native diet and had a strong influence on household food consumption, while younger individuals were more likely to adapt to a western lifestyle [24]. This could mean that the acculturation process might be more challenging for those who are older when migrating. The argumentation concludes that the question regarding if, how, and why older persons change their food habits and the relationship to health when migrating remains to be investigated.

Almost seven out of ten participants rated their health as good. This result is well in line with a Swedish report [25] from 2016 stating that two-thirds of persons 65-74 years rate their health as good, even though the same report also states that the proportion decreases with higher age. Our finding with significant difference between genders is also in line with previous findings $[6,26,27]$ stating a gender difference where older women rate their health lower than men in the same age. However, data on older immigrants' self-rated health in relation to food habits in Sweden is sparse, making the contextualisation of our findings difficult. Even so, results from earlier studies are diverse. We found only one study [28] showing that self-rated health status among nativeand foreign-born persons did not differ. Instead, those who were younger, married, non-smokers with a higher income were less likely to rank their health as poor. Another study indicates that some immigrant groups may be at higher risk for non-communicable diseases caused by obesity and inadequate physical activity [29], and show more signs of distress than native-born people [30]. Data from Gothenburg municipality shows that the proportion of persons (all ages) in the primary urban district of PAMC, who consider their health as poor, is somewhat higher compared to Gothenburg as a whole [31]. The level of education and income in the area is lower, and the sickness rate higher than the general level in the city [32]. Previous research has shown that people with low socioeconomic background are often underrepresented or excluded in scientific studies [33], further stressing the need for including a larger, more varied study sample for increased understandings and generalisability in the field of food habits. A sample more heterogeneous in terms of diet and health may have given more power to determine true associations between meal frequency, vegetable intake and self-rated health. As no clinical information on health status was asked for, this issue is not possible to address but would have been interesting as several health conditions could be expected to be prevalent in this age group.

\section{Strengths and limitations}

A strength of this study is the possibility of studying a relatively unexplored area of immigrant research with the main advantage of being able to generate future 
research questions. However, this study contains secondary analyses of data from a health-promoting intervention aimed at older immigrants, an approach with several limitations.

Food habits, operationalised as meal frequency and vegetable intake, were found not to be associated with self-rated health or life satisfaction in our study. The selfreported dietary data for the examination of meal frequency and vegetable intake is crude, and could recall bias. Unfortunately, the quality of the meals was not asked for, this could have increased the validity of the study. Adjustments for confounders did not affect the results. One simple explanation for this is, consequently, that no such association exists. However, there are important methodological aspects that need to be considered, as one of the disadvantages of a secondary analysis is that the design of the original study is set and not changeable. In this study, we have chosen to dichotomise the variables. The result becomes clearer and more concise while dichotomising, but on the other hand, nuances in data will be erased. In addition, the sample is small and using the whole scale would have meant that there would have been too few individuals in each scale step.

According to the assessment of life satisfaction in PAMC, a modified version containing eight out of the original eleven items in LiSat-11 was used. The delimitation of items was motivated by the reference groups, which constituted part of the participatory approach implemented in PAMC [14]. Even if the clearly elaborated participatory approach in PAMC was a strength in many respects, this proceeding changed the psychometric characteristics of the instrument, and thereby, the reliability and validity of the modified version can be questioned. However, it is a balancing act to, on the one hand, adhere to one method and, on the other hand, to respect cultural differences in order to reach a higher level of participation.

The question regarding vegetable intake did not specify the type or amount of consumed vegetables, a limitation for the study as it was left to the participant to decide what he or she included in the concept "vegetables". A predefined definition would have made it possible to separate vegetables from root vegetables and fruit in order to achieve complete information. However, the assessment only asked if the participants ate vegetables or not, limiting the opportunities to deepen our knowledge of older immigrants' vegetable intake. In addition, the use of set response alternatives might have limited the participants to certain answers. Also, the content validity [34] of the questions regarding meals (e.g. breakfast, lunch, dinner and snack) is general and does not admit analysis of the composition of the meals, also leaving it to be freely interpreted by the research assistant. To ensure that valid and reliable instruments and questions are used, we recommend that a dietician or a nutrition-trained researcher should be part of the research group planning future studies in the field to help create more accurate dietary intake instruments.

There are also considerations according to the study sample which was small. Age at the time of immigration as well as education and gender affects how rapidly a person born abroad is integrated in the recipient country [25]. Therefore, a larger study sample representing persons with a broader range of years living in Sweden and a variety of country of birth would be of great value in future studies of food habits among immigrants. However, expanding the inclusion criteria by inviting people regardless of country of birth in a future study will involve some practical difficulties such as the need for interpreters and project assistants with additional language skills. Including an adequate number of people from different countries of birth during a study period can make it difficult to reach a sufficiently large number of participants to be able to carry out subgroups analyses, as these will be too small and further limiting the study. Moreover, language in itself could be a methodological challenge; language barriers are one common reason for persons not to participate in research [33]. A strength in PAMC was that a bilingual approach was applied. The questionnaire was translated and the participants could choose their preferred language for the interviews. In addition, project assistants sharing the participants' mother tongue were involved in the compilation of data, contributing to the quality of the results. Another approach trying to overcome the language barrier could be to use images of food as a supplement. Such an approach would also allow illiterate older immigrants to participate in the study, a promising strategy to prevent selection bias.

Finally, the secondary analysis was performed without regard to participant's country of birth, as the intention never was to analyse subgroups. One reason was that forming subgroups based on country of birth would result in too small groups for accurate analysis. In addition, there is heterogeneity within each subgroup that might be of more importance than the country of birth, for example, reason for migration or socioeconomic situation.

Since there is little research concerning immigrants and health related factors such as their food habits, future studies might consider a mixed method design combining qualitative and quantitative methods [35]. First, qualitative interviews could be carried out with a small group of immigrants who share the same culture to get an idea of the group's dietary habits in order to use this knowledge to create an interview guide with questions relevant to the group. Then, based on the findings of these interviews, 
structured interviews could be carried out with a much larger sample. To obtain more detailed information regarding present food habits, the use of a well-established dietary intake method is also recommended [36].

To sum up, data on health and food habits for older immigrants in Sweden are sparse and evasive. Hence, baseline measures in PAMC offered a unique opportunity to explore the research questions. The results point to the fact that future studies of food habits of older immigrants in Sweden need an improved study design. However, secondary analysis involves methodological problems which affect the interpretation of results. In future research, several considerations could be taken into account; a broader range of years living in Sweden, immigrants' selfrated health in the light of demographical factors such as age, gender and education and the fact that the conditions concerning both the reason for migration and the acculturation process vary depending on several different factors. Our study offers suggestions for such improvements and the results form a basis for new research questions regarding food habits in the target group.

\section{Conclusion}

The result shows that almost half of older adults, 70 years and older, who have migrated to Sweden from Finland or the Western Balkan region have changed their food habits and about one-fifth miss specific food from their native country. It also showed that there was a significant difference in self-rated health in favour of the male participants in PAMC, but that food habits, operationalised as meal frequency or vegetable intake, were not associated with self-rated health or life satisfaction. Nevertheless, it is still important to maintain good food habits in old age in order to retain a healthy ageing. The growing inequality in health among foreign-born people compared to the general Swedish population calls for development of policy recommendations specifically aimed at different ethnic groups. However, the results were affected by methodological shortcomings which, nonetheless, indicate a number of directions in which the design can be improved. Future studies on food habits as an important lifestyle factor potentially affecting health of older immigrants are therefore needed since it is an increasing group in most European societies.

\section{Supplementary Information}

The online version contains supplementary material available at https://doi. org/10.1186/s42779-021-00115-2.

Additional file 1. Relationship between meal frequency and self-rated health. Meals not dichotomized. Relationship between meal frequency life satisfaction. Meals not dichotomized.
Additional file 2. Relationship between vegetable intake and self-rated health. Vegetables not dichotomized. Relationship between vegetable intake and life satisfaction. Vegetables not dichotomized.

\section{Acknowledgements}

The authors would like to thank Jennifer Herriman for proof reading the manuscript

\section{Authors' contributions}

JJ was primarily responsible for drafting the manuscript and performing the data analyses. ER, FS and SG were involved in designing the secondary analysis of the original study, drafting the manuscript and contributing to critical revisions during the writing process. All authors read and approved the final manuscript.

\section{Funding}

This research received funding from the Swedish National Graduate School for Competitive Science on Ageing and Health (SWEAH) and The Vårdal Institute. SWEAH and The Varrdal Institute funded the first authors PhD study. The funding bodies had no involvement in this study and did not influence the study design, collection, analysis and interpretation of data, nor the writing of this paper.

Availability of data and materials

The dataset used and analysed during the current study is available from the corresponding author on reasonable request.

\section{Declarations}

\section{Ethics approval and consent to participate}

The Regional Ethical Review Board in Gothenburg approved PAMC (\#821-11). Written informed consent was obtained from all participants. All participants received written and oral information that participation was completely voluntary and that they could decline at any time without giving a reason. The PAMC was registered at ClinicalTrials.gov April 10, 2013, identifier: NCT01841853

\section{Consent for publication}

Not applicable.

\section{Competing interests}

The authors declare that they have no competing interests.

\section{Author details}

${ }^{1}$ Department of Internal Medicine and Clinical Nutrition, Sahlgrenska Academy at University of Gothenburg, P.O. Box 459, 40530 Gothenburg, Sweden. ${ }^{2}$ Department of Health Sciences, Swedish National Graduate School for Competitive Science on Ageing and Health (SWEAH), Lund University, P.O. Box 157, 22100 Lund, Sweden. ${ }^{3}$ Centre for Ageing and Health (AgeCap), University of Gothenburg, Wallinsgatan 6, 43141 Mölndal, Sweden. ${ }^{4}$ Department of Rehabilitation, School of Health and Welfare, Jönköping University, Jönköping, Sweden. ${ }^{5}$ Department of Food and Nutrition and Sports Science, University of Gothenburg, P.O Box 300, 40530 Gothenburg, Sweden. ${ }^{6}$ Faculty of Health Science, Kristianstad University, 29188 Kristianstad, Sweden.

Received: 1 February 2021 Accepted: 22 November 2021

Published online: 07 December 2021

References

1. SCB [Statistics Sweden]. The future population of Sweden 2015-2060. 2015

2. SCB [Statistics Sweden]. Statistics Database [Internet]. 2019 [cited 2020 Nov 2]. http://www.statistikdatabasen.scb.se/pxweb/sv/ssd/START BE_BE0101_BE0101E/FodelselandArK/?rxid=f45f90b6-7345-4877ba25-9b43e6c6e299. 
3. Koctürk-Runefors T. Changes in food habits and nutritional status of immigrants from Turkey in Sweden. Nutr Adapt New Life-Styles. 1990:45:157-64.

4. Satia-Abouta J. Dietary acculturation: definition, process, assessment, and implications. Int J Hum Ecol. 2003;4(1):71-86.

5. Koochek A, Mirmiran P, Sundquist K, Hosseini F, Azizi T, Moeini A, et al. Dietary differences between elderly Iranians living in Sweden and Iran a cross-sectional comparative study. BMC Public Health. 2011;11(411):1-8.

6. Koochek A, Montazeri A, Johansson S, Sundquist J. Health-related quality of life and migration: a cross-sectional study on elderly Iranians in Sweden. Health Qual Life Outcomes. 2007:5(60):1-8.

7. Ocampo J. Self-rated health: importance of use in elderly adults. Colomb Med. 2010;41(3):275-89.

8. Hall A. Concept of life satisfaction. In: Encyclopedia of quality of life and well-being research; 2014. p. 3599-601.

9. Bowling A, Farquhar M, Grundy E, Formby J. Changes in life satisfaction over a two and a half year period among very elderly people living in London. Soc Sci Med. 1993;36(5):641-55.

10. André B, Canhão H, Espnes G, Rodrigues A, Gregorio M, Nguyen C, et al. Is there an association between food patterns and life satisfaction among Norway's inhabitants ages 65 years and older? Appetite. 2017;110:108-15.

11. The city of Gotenburg. Jämlikhetsrapporten 2017 [In Swedish]. 2017.

12. Johannesson J, Rothenberg E, Dahlin-Ivanoff S, Slinde F. Gender differences in practice: knowledge and attitudes regarding food habits and meal patterns among community dwelling older adults. J Aging Res Clin Pract. 2016;5(4):220-8.

13. Dahlin-Ivanoff $S$, Gosman-Hedström G, Edberg A-K, Wilhelmson K, Eklund $\mathrm{K}$, Duner $\mathrm{A}$, et al. Elderly persons in the risk zone. Design of a multidimensional, health-promoting, randomised three-armed controlled trial for "prefrail" people of $80+$ years living at home. BMC Geriatr. 2010;10(27):1-10

14. Gustafsson S, Lood Q, Wilhelmsson K, Häggblom-Kronlöf G, Landahl S, Dahlin-Ivanoff S, et al. A person-centred approach to health promotion for persons 70+ who have migrated to Sweden: Promoting Aging Migrants' Capabilities implementation and RCT study protocol. BMC Geriatr. 2015;15(1):10

15. Gustafsson S, Wilhelmson K, Eklund K, Gosman-Hedström G, Zidén L, Kronlöf G, et al. Health-promoting interventions for persons aged 80 and older are successful in the short term-results from the randomized and three-armed Elderly Persons in the Risk Zone study. J Am Geriatr Soc. 2012:60(3):447-54.

16. Stebbins RA. Exploratory research in the social sciences. London: Sage Publications; 2001.

17. Folstein M, Folstein S, McHugh P. "Mini-mental state": a practical method for grading the cognitive state of patients for the clinician. J Psychiatr Res. 1975;12(3):189-98.

18. Ware J, Sherbourne C. The MOS 36-item short-form health survey (SF-36): I. Conceptual framework and item selection. Med Care. 1992;30(6):473-83.

19. Fugl-Meyer A, Bränholm I, Fugl-Meyer K. Happiness and domain-specific life satisfaction in adult northern Swedes. Clin Rehabil. 1991;5(1):25-33.

20. Guba E. Criteria for assessing the trustworthiness of naturalistic inquiries. Educ Technol Res Dev. 1981;29(2):75-91.

21. Samuelsson J, Rothenberg E, Lissner L, Eiben G, Zettergren ASI. Time trends in nutrient intake and dietary patterns among five birth cohorts of 70-year-olds examined 1971-2016: results from the Gothenburg H70 birth cohort studies, Sweden. Nutr J. 2019;18(66):1-17.

22. Ore H. Ambivalent nostalgia: Jewish-Israeli migrant women "cooking" ways to return home. Food Cult Soc. 2018;21(4):568-84

23. Duruz J. Living in Singapore, travelling to Hong Kong, remembering Australia: intersections of food and place. J Aust Stud. 2006;30(87):110-5.

24. Satia-Abouta J, Patterson R, Kristal A, Teh C, Tu S. Psychosocial predictors of diet and acculturation in Chinese American and Chinese Canadian women. Ethn Health. 2002;7(1):21-39.

25. Gustafsson BA, Mac Innes H, Österberg T. Age at immigration matters for labor market integration-the Swedish example. IZA J Dev Migr. 2017;7(1):1.

26. Lennartsson C, Agahi N, Wånell S. Äldre personers hälsa och livssituation. Stockholm; 2012.
27. Folkhälsomyndigheten. Självskattat allmänt hälsotillstånd [Internet]. 2017 [cited 2020 Nov 2]. Available from: https://www.folkhalsomyndigheten. se/folkhalsorapportering-statistik/folkhalsans-utveckling/halsa/sjalvskatt at-allmant-halsotillstand/.

28. Newbold K. Self-rated health within the Canadian immigrant population: risk and the healthy immigrant effect. Soc Sci Med. 2005;60:1359-70.

29. Rechel B, Mladovsky P, Ingleby D, Mackenbach J. Migration and health in an increasingly diverse Europe. Lancet. 2013;381(9873):1235-45.

30. Johnson C, Rostila M, Svensson A, Engström K. The role of social capital in explaining mental health inequalities between immigrants and Swedishborn: a population-based cross-sectional study. BMC Public Health. 2017;17(117):1-15.

31. Göteborgs Stad (The city of Gotenburg). Hälsobladet 2019 [Internet]. 2019 [cited 2020 Nov 2]. p. 12. https://goteborg.se/wps/portal/enhet ssida/statistik-och-analys?uri=gbglnk\%3A201836103354857.

32. The City of Gothenburg. SDN Presentations 2017 [Internet]. 2017 [cited 2020 Aug 5]. http://statistik.goteborg.se/Statistik/Presentationer/SDNPresentationer-2017/.

33. Hussain Gambles M, Atkin K, Leese B. Why ethnic minority groups are underrepresented in clinical trials: a review of the literature. Health $\mathrm{Soc}$ Care Community. 2004;12(5):382-8.

34. Polit DF, Beck CT. The content validity index: are you sure you know what's being reported? Critique and recommendations. Res Nurs Health. 2006;29(5):489-97.

35. Creswell J, Creswell D. Research design: qualitative, quantitative and mixed methods approaches. 5th ed. Los Angeles: SAGE Publications Ltd; 2018.

36. Creswell J, Plano CV. Designing and conducting mixed methods research 2nd ed. London: SAGE Publications Ltd; 2011.

\section{Publisher's Note}

Springer Nature remains neutral with regard to jurisdictional claims in published maps and institutional affiliations.

Ready to submit your research? Choose BMC and benefit from

- fast, convenient online submission

- thorough peer review by experienced researchers in your field

- rapid publication on acceptance

- support for research data, including large and complex data types

- gold Open Access which fosters wider collaboration and increased citations

- maximum visibility for your research: over 100M website views per year

At $\mathrm{BMC}$, research is always in progress.

Learn more biomedcentral.com/submissions 